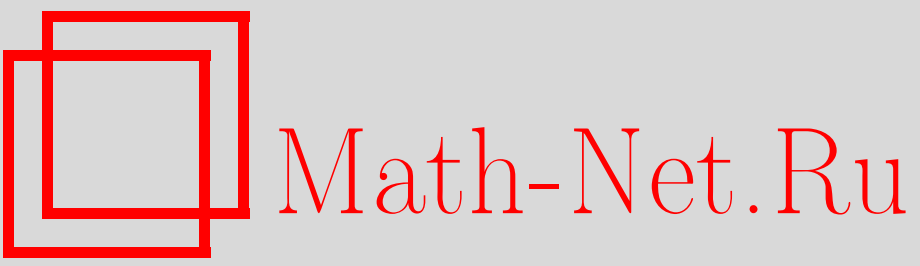

В. М. Круглов, Простое доказательство неограниченности однородных случайных процессов с независимыми приращениями, Теория вероятн. и ее примен., 2011, том 56, выпуск 4, 806-808

DOI: https://doi.org/10.4213/tvp4425

Использование Общероссийского математического портала Math-Net.Ru подразумевает, что вы прочитали и согласны с пользовательским соглашением

http://www.mathnet.ru/rus/agreement

Параметры загрузки:

IP : 54.198 .55 .26

26 апреля 2023 г., 13:05:45 


\title{
ПРОСТОЕ ДОКАЗАТЕЛЬСТВО НЕОГРАНИЧЕННОСТИ ОДНОРОДНЫХ СЛУЧАЙНЫХ ПРОЦЕССОВ С НЕЗАВИСИМЫМИ ПРИРАЩЕНИЯМИ
}

\begin{abstract}
В настоящей заметке доказано, что неограниченность непостоянных однородных случайные процессов с независимыми приращениями является естественным следствием закона нуля или единицы Колмогорова. Известное доказательство этого утверждения основано на глубоком предварительном исследовании случайных процессов с независимыми приращениями.
\end{abstract}

Ключевые слова и фразы: закон нуля или единицы Колмогорова, случайные процессы с независимыми приращениями.

Предполагается, что все рассматриваемые далее случайные процессы определены на вероятностном пространстве $(\Omega, \mathscr{F}, \mathbf{P})$ и принимают свои значения в конечномерном евклидовом пространстве $\mathbf{R}^{d}$.

Напомним, что случайный процесс $\left\{X_{t}, t \geqslant 0\right\}$ имеет независимье приращения, если для любых чисел $n \in \mathbf{N}=\{1,2, \ldots\}, n \geqslant 2$, и $0 \leqslant t_{1}<t_{2}<\cdots<t_{n}$ случайные векторы $X_{t_{1}}, X_{t_{2}}-X_{t_{1}}, \ldots, X_{t_{n}}-X_{t_{n-1}}$ независимы. Случайный процесс $\left\{X_{t}, t \geqslant 0\right\}$ называется однороднылм, если для любых чисел $s, t, 0 \leqslant s<t, h>0$ приращения $X_{t+h}-X_{s+h}$ и $X_{t}-X_{s}$ одинаково распределены.

Заметим, что каждая из $d$ координат однородного случайного процесса $\left\{X_{t}, t \in\right.$ $T\}$ является однородным вещественным случайным процессом. В этом легко убедиться с помощью характеристических функций. Обозначим $\langle a, b\rangle$ скалярное произведение $a, b \in \mathbf{R}^{d}$. Для любых чисел $s, t, 0 \leqslant s<t, h>0, u \in \mathbf{R}$ и для любого $v \in \mathbf{R}^{d}$ выполняются равенства

$$
\mathbf{E} e^{i u\left(\left\langle v, X_{t+h}\right\rangle-\left\langle v, X_{s+h}\right\rangle\right)}=\mathbf{E} e^{i\left(\left\langle u v, X_{t+h}-X_{s+h}\right\rangle\right)}=\mathbf{E} e^{i\left(\left\langle u v, X_{t}-X_{s}\right\rangle\right)}=\mathbf{E} e^{i u\left(\left\langle v, X_{t}\right\rangle-\left\langle v, X_{s}\right\rangle\right)} .
$$

Равенство функций аргумента $u \in \mathbf{R}$ слева и справа означает, что вещественный случайный процесс $\left\{\left\langle v, X_{t}\right\rangle, t \geqslant 0\right\}$ обладает свойством однородности. Среди всех случайных процессов с независимыми приращениями мы выделим те, которые не представляют для нас особого интереса. Случайный процесс $\left\{X_{t}, t \geqslant 0\right\}$ называется постоянныл, если $X_{t}(\omega)=c$ для всех $t \geqslant 0$, для некоторого $c \in \mathbf{R}^{d}$ и для почти всех $\omega \in \Omega$.

Известно (см. [1, с. 139]), что любой вещественный непостоянный однородный случайный процесс с независимыми приращениями неограничен. Приведенное в [1] доказательство построено на глубоком предварительном исследовании случайных процессов с независимыми приращениями. В настоящей заметке доказано, что это утверждение вытекает из закона нуля или единицы Колмогорова.

Теорема. Любой непостоянньй однородный случайный прочесс $\left\{X_{t}, t \geqslant 0\right\} c$ независимьми приращениями почти всюду неограничен, т.е. $\sup _{t \geqslant 0}\left|X_{t}\right|=\infty$ почти всюду.

Д о к а з а т е л ь с т в о. Теорему достаточно доказать для какой-либо координаты случайного процесса. Далее предполагается, что $\left\{X_{t}, t \geqslant 0\right\}$ является непостоянным вещественным случайным процессом с независимыми приращениями. Мы

* Московский государственный университет им. М. В. Ломоносова, факультет вычислительной математики и кибернетики, Ленинские горы, 119991 Москва, Россия; e-mail: krugvictor@gmail.com

1) Работа выполнена при поддержке РФФИ, грант 11-01-00515-а. 
будем предполагать, что $X_{0}=0$. Это условие не ограничивает общность рассуждений. Случайные величины $X_{n}-X_{n-1}, n \in \mathbf{N}$, невырождены, независимы и одинаково распределены. В силу неравенства $\sup _{0 \leqslant t<\infty}\left|X_{t}\right| \geqslant \sup _{k \in \mathbf{N}}\left|X_{k}\right|$ достаточно доказать, что $\sup _{k \in \mathbf{N}}\left|X_{k}\right|=\infty$ почти всюду. Так как $\left|X_{n}\right|<\infty$ для любого $n \in \mathbf{N}$, то справедливо равенство $\left\{\sup _{k \in \mathbf{N}}\left|X_{k}\right|=\infty\right\}=\left\{\sup _{\mathbf{N} \ni k>n}\left|X_{k}-X_{n}\right|=\infty\right\}$. Мы намерены воспользоваться законом нуля или единицы Колмогорова (см. $[2$, с. 116]). Обозначим $\mathscr{F}_{n}$ сигма-алгебру, порожденную случайными величинами $X_{k}-X_{k-1}$, $k \geqslant n$. Событие $A=\left\{\sup _{k \in \mathbf{N}}\left|X_{k}\right|=\infty\right\}$ можно представить в виде пересечения $A=\cap_{r=1}^{\infty}\left\{\sup _{\mathrm{N} \ni k>n}\left|X_{k}-X_{n}\right|>r\right\}$ событий из сигма-алгебры $\mathscr{F}_{n}$. Поэтому $A \in \mathscr{F}_{n}$ для любого $n \in \mathbf{N}$ и, следовательно, $A \in \cap_{n=1}^{\infty} \mathscr{F}_{n}$. По упомянутому закону нуля или единицы вероятность события $A$ равна нулю или единице.

Докажем, что $\mathbf{P}\{A\}=1$. Предположим противное, что $\mathbf{P}\{A\}=0$. Поэтому $\sup _{k \in \mathbf{N}}\left|X_{k}\right|<\infty$ почти всюду (п.в.) и, следовательно,

$$
\lim _{n \rightarrow \infty} \frac{1}{n^{1 / m}} \sum_{k=1}^{n}\left(X_{k}-X_{k-1}\right)=\lim _{n \rightarrow \infty} \frac{X_{n}}{n^{1 / m}}=0 \quad \text { п.в. }
$$

для любого $m \in \mathbf{N}$. Отсюда следует, что

$$
\lim _{n \rightarrow \infty} \frac{X_{n}-X_{n-1}}{n^{1 / m}}=\lim _{n \rightarrow \infty} \frac{X_{n}}{n^{1 / m}}-\lim _{n \rightarrow \infty} \frac{(n-1)^{1 / m}}{n^{1 / m}} \frac{X_{n-1}}{(n-1)^{1 / m}}=0
$$

п.в. По лемме Бореля-Кантелли ряд $\sum_{n=1}^{\infty} \mathbf{P}\left\{\left|X_{n}-X_{n-1}\right|>n^{1 / m}\right\}$ сходится. Случайные величины $X_{n}-X_{n-1}, n \in \mathbf{N}$, одинаково распределены и, следовательно,

$$
\sum_{n=1}^{\infty} \mathbf{P}\left\{\left|X_{n}-X_{n-1}\right|>n^{1 / m}\right\}=\sum_{n=1}^{\infty} \mathbf{P}\left\{\left|X_{1}\right|>n^{1 / m}\right\} .
$$

Известно, что сходимость последнего ряда влечет конечность математического ожидания $\mathbf{E}\left|X_{1}\right|^{m}$. Действительно,

$$
\begin{aligned}
\mathbf{E}\left|X_{1}\right|^{m} & =\sum_{n=1}^{\infty} \mathbf{E}\left(\left|X_{1}\right|^{m} I_{\left\{n-1<\left|X_{1}\right|^{m} \leqslant n\right\}} \leqslant \sum_{n=1}^{\infty} n \mathbf{P}\left\{n-1<\left|X_{1}\right|^{m} \leqslant n\right\}\right. \\
& =\sum_{n=1}^{\infty} n\left(\mathbf{P}\left\{\left|X_{1}\right|^{m}>n-1\right\}-\mathbf{P}\left\{\left|X_{1}\right|^{m}>n\right\}\right) \\
& =\sum_{n=0}^{\infty} \mathbf{P}\left\{\left|X_{1}\right|^{m}>n\right\}<\infty
\end{aligned}
$$

Если $\mathbf{E} X_{1} \neq 0$, то $\lim _{n \rightarrow \infty}\left(X_{n} / n\right)=\mathbf{E} X_{1}$ п.в. по усиленному закону больших чисел Колмогорова. Отсюда следует, что $\sup _{n \in \mathbf{N}}\left|X_{n}\right|=\infty$ п.в. Это противоречит предположению, что $\mathbf{P}\{A\}=0$. Если $\mathbf{E} X_{1}=0$, то по центральной предельной теореме мы получим

$$
\lim _{n \rightarrow \infty} \mathbf{P}\left\{\frac{X_{n}}{\sqrt{n \mathbf{E}\left|X_{1}\right|^{2}}}<x\right\}=\frac{1}{\sqrt{2 \pi}} \int_{-\infty}^{x} e^{-u^{2} / 2} d u
$$

для любого $x \in \mathbf{R}$. С другой стороны,

$$
\lim _{n \rightarrow \infty} \mathbf{P}\left\{\frac{X_{n}}{\sqrt{n \mathbf{E}\left|X_{1}\right|^{2}}}<x\right\}= \begin{cases}1, & \text { если } x>0 \\ 0, & \text { если } x<0 .\end{cases}
$$

Мы снова пришли к противоречию и, следовательно, $\mathbf{P}\{A\}=1$. Теорема доказана. 


\section{СПИСОК ЛИТЕРАТУРЫ}

1. Скороход A. В. Случайные процессы с независимыми приращениями. М.: Наука, $1964,278 \mathrm{c}$.

2. Колмогоров A.В. Основные понятия теории вероятностей. 2-е изд. М.: Наука, $1974,119 \mathrm{c}$.

\section{УТОЧНЕНИЕ ОСТАТОЧНОГО ЧЛЕНА В ТЕОРЕМЕ ЛЯПУНОВА}

Доказаны новые оценки близости характеристических функций нормированных сумм независимых случайных величин к характеристической функции стандартного нормального закона, что позволило эффективно оценить скорость сходимости в центральной предельной теореме при наличии абсолютных моментов третьего порядка. А именно, установлено, что константа $C$, фигурирующая в неравенстве Берри-Эссеена, не превосходит 0.4774. Кроме того, показано, что константа, возникающая в аналогичном неравенстве для неодинаково распределенных слагаемых, не превосходит 0.5591 .

Ключевые слова и фразы: центральная предельная теорема, теорема Ляпунова, скорость сходимости, неравенство Берри-Эссеена.

Рассмотрим независимые (действительные) невырожденные случайные величины (с.в.) $X_{1}, \ldots, X_{n}$ с нулевыми средними, дисперсиями равными $\sigma_{1}^{2}, \ldots, \sigma_{n}^{2}$ и конечными третьими абсолютными моментами $\beta_{1}, \ldots, \beta_{n}$. Обозначим

$$
\sigma^{2}=\sigma^{2}(n):=\sum_{j=1}^{n} \sigma_{j}^{2}, \quad \varepsilon_{n}:=\frac{1}{\sigma^{3}} \sum_{j=1}^{n} \beta_{j} .
$$

Величина $\varepsilon_{n}$ называется дробью Ляпунова и позволяет эффективно оценить скорость сходимости в центральной предельной теореме при наличии моментов третьего порядка. Согласно теореме Ляпунова $S_{n}:=\left(X_{1}+\cdots+X_{n}\right) / \sigma(n)$ слабо сходится к стандартной нормальной с.в. при $\varepsilon_{n} \rightarrow 0$. Более того, известно (см., например, [1]), что существует наименьшая универсальная постоянная $C$, такая, что для расстояния Колмогорова между $S_{n}$ и стандартной гауссовской величиной $N$ справедливо неравенство

$$
\rho\left(S_{n}, N\right):=\sup _{x \in \mathbf{R}}\left|\mathbf{P}\left(S_{n} \leqslant x\right)-\mathbf{P}(N \leqslant x)\right| \leqslant C \varepsilon_{n}, \quad n \in \mathbf{N} .
$$

Оценке этой константы посвящено множество работ. Первый результат в этой области принадлежит К. Эссеену [1], который в 1942 г. показал, что $C \leqslant 7.5$. За 40 лет усилиями Г. Бергстрема, К. Такано, В. М. Золотарева, П. ван Бика, И. С. Шиганова и

* Московский государственный университет им. М.В.Ломоносова, механикоматематический факультет, кафедра теории вероятностей, Ленинские горы, 119991 Москва, Россия; e-mail: itiurin@gmail.com

1) Работа выполнена при финансовой поддержке Фонда поддержки молодых ученых «Конкурс Мебиуса», а также Российского фонда фундаментальных исследований, проект 10-01-00397а. 